\title{
ASSESSMENT OF MEDICATION EFFICACY ON DETERMINANTS OF PSYCHIATRIC ILLNESS AMONG DIABETES MELLITUS PARTICIPANTS
}

\author{
Akza K Alex*, Bharathi DR, Nataraj GR, Adarsh Mathew, Tejaswini SM \\ SJM College of Pharmacy, Chitradurga-577502
}

Article Info: Received 20 April 2019; Accepted 17 May. 2019

DOI: https://doi.org/10.32553/jbpr.v8i3.597

Address for Correspondence: Akza K Alex, M.Pharm Student, SJM College of Pharmacy, Chitradurga

Conflict of interest statement: No conflict of interest

\section{ABSTRACT:}

Introduction: The prevalence of Diabetes Mellitus (DM) among adults has been increased considerably across the globe, and has contributed a substantial proportion in annual mortality rate. Diabetes and psychiatric disorders are having such an interface that they both influence each other in several ways.

Objectives: The study was conducted to assess the prevalence, determinant factors, prescription pattern, efficacy and adverse reactions in diabetic patients associated with psychiatric illness in a tertiary care hospital.

Materials and Methods: A prospective observational study was carried out for a period of ten months in General Medicine and Psychiatric Department, Basaveswara Medical College Hospital and Research centre, Chitradurga.

Results: In this study, a total of 101 diabetic patients with or without co-morbidities were enrolled in the study. Among them, 27 in-patients were associated with psychiatric illness which was considered as comorbid condition. The prevalence of psychiatric illness with diabetes mellitus was found to be $26.7 \%$ which is highly significant with the p-value (0.00). A higher prevalence was noticed with age group 41-50 years and with male gender. The common determinant factors of psychiatric illness in diabetic patients were being women, having no income, being at the age of menopause, having no physical activity, being illiterate and being single or widowed. Among the anti-diabetic drug prescriptions, there was a higher percentage of use of insulin and the most commonly prescribed drug was combination of Glimepride + Metformin (sulfonylurea and biguanide). The most commonly prescribed anti-psychotic drugs were chlordiazepoxide and alprazolam. Conclusion: The study reveals a high prevalence of psychiatric illness in diabetic patients with determinant factors of psychiatric illness in diabetic patients were being women, having no income, being at the age of menopause, having no physical activity, being illiterate and being single or widowed.

Key Words: Prevalence; Determinant factors; Diabetes mellitus; Psychiatric illness

\section{INTRODUCTION}

The association between DM and psychiatric disorders has been a captivating issue for endocrinologists and mental health professionals for several years. Sir Henry Maudsley has mentioned that "Diabetes is a disease which often shows itself in families in which insanity prevails". Diabetes and psychiatric disorders are having such an interface that they both influence each other in several ways. ${ }^{1}$
According to reports, the decisive factors or determinant factors for the interdependence between psychiatric disorders and DM are lack of education, lower income, low socio-economic status, being single, older age and long duration of follow-up. ${ }^{2}$ Assorted features may be responsible for the amplified predisposition to develop DM in psychiatric patients. They may be factors such as use of anti-psychotic drugs, poor health behaviors, stress, lack of physical activity, heritability, poor dietary habits etc. ${ }^{3}$ 
This study is majorly accomplished to further elucidate and to ascertain the interconnection of psychiatric illness and DM by monitoring prevalence rates and determinant factors of psychiatric illness among DM patients. This study also intended at evaluating the medication efficacy as well as possible adverse drug reactions (ADRs) among these patients.

\section{MATERIALS AND METHODS}

Study Site: The study has been conducted in General Medicine and Psychiatric Department, Basaveswara Medical College Hospital and Research centre, Chitradurga.

Study design: It is a prospective observational study.

Study period: The study was conducted for a period of ten months from 2018 to 2019.

Study subjects: The study included the subjects who meet the following criteria

\section{Inclusion Criteria:}

- $\quad$ Both inpatients and out patients.

- Both genders.

- Diabetic patients with psychiatric disorders.

- Patients having type I and type-II Diabetes Mellitus.

\section{Exclusion Criteria:}

- Comatose subjects.

Sources of data:

- Demographics, medication and medical history were collected from the patient profile form and their prescriptions.

\section{Study procedure:}

The study was initiated after obtaining the ethical clearance from Institutional Ethics Committee
(IEC). Subjects who have satisfied the above study criteria have been recruited into the study. All the subjects were explained regarding the study and Informed Consent Form was obtained. The demographic details, medical information, medication data and other relevant information were documented in a pre structured data collection form. A self-designed questionnaire was used to assess the determinant factors and the quality of life of the subjects.

\section{Statistical Analysis}

The data were entered in Microsoft Excel sheets and analysis has been done by Social Programme Scientific Software (SPSS) version 24. The data was analyzed by Descriptive methods (Mean), onesample t test and Chi-square test for correlation.

\section{RESULTS}

A total of 101 diabetic patients with or without comorbid were enrolled in the study. Among them, 27 in-patients were associated with psychiatric illness which was considered as co-morbid condition. Further, the data of 27 diabetic patients associated with psychiatric illness were analyzed and presented as follows,

\section{Prevalence of Various Psychiatric Illnesses among Diabetic Patients}

The results of prevalence of psychiatric illnesses among diabetic patients are as follows: Among the 27 subjects, 12(11.87\%) were suffering from anxiety with DM, followed by $5(4.94 \%)$ were suffering from dementia with DM, 5 (4.94\%) were suffering from seizure with DM, 4(3.96\%) were suffering from psychosis with DM and $1(0.99 \%)$ was suffering from schizophrenia with DM. Results were shown in table no.1 and graphically represented in fig.no.1

Table 1: Distribution of various psychiatric illnesses among diabetic patients $(n=27)$

\begin{tabular}{|l|l|l|}
\hline Psychiatry illness Status & Frequency & Prevalence (\%) \\
\hline DM with Anxiety & 12 & 11.87 \\
\hline DM with Psychosis & 04 & 3.96 \\
\hline DM with Dementia & 05 & 4.94 \\
\hline DM with Seizure & 05 & 4.94 \\
\hline DM with Schizophrenia & 01 & 0.99 \\
\hline Total & $\mathbf{2 7}$ & $\mathbf{2 6 . 7}$ \\
\hline
\end{tabular}




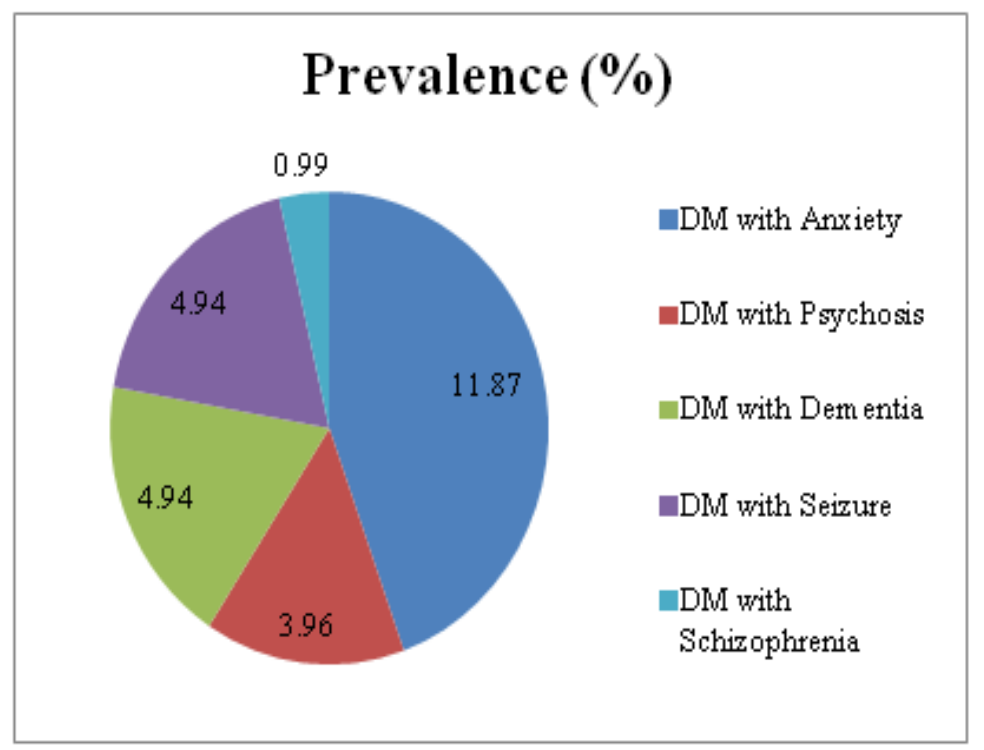

Figure 1: Distribution of various psychiatric illnesses among diabetic patients

\section{Determinant Factors Associated With Diabetes Mellitus and Psychiatric Illness}

Determinant factors responsible for psychiatric illness associated with DM were considered to be, being women, having no income, being single or widowed, being at the age of menopause, having no physical activity and being illiterate. The results were shown in table no. 2 .

Table 2: Determinant factors associated with diabetes mellitus and psychiatric illness

\begin{tabular}{|l|l|l|}
\hline Determinant factor & Frequency & $\begin{array}{l}\text { Percentage } \\
\text { (\%) }\end{array}$ \\
\hline Being women & 12 & 44.4 \\
\hline No income & 10 & 37.1 \\
\hline Widow and unmarried & 02 & 7.4 \\
\hline Menopause (n=12) & 07 & 25.9 \\
\hline No physical activity & 06 & 22.2 \\
\hline Illiterate & 05 & 18.5 \\
\hline
\end{tabular}

\section{Prescription Pattern of Drugs}

In 27 prescriptions, 61 drugs were prescribed and the average drugs per prescription were found to be 2.2. Among 61 drugs prescribed, 32 were anti- diabetic drugs and 29 were psychiatric drugs and out of that 32 anti-diabetic drugs, 18 (29.5\%) were insulin and 14 were oral hypoglycaemic. Amongst the 14 oral hypoglycaemics, the most commonly prescribed drug was found to be combination of Glimepiride+Metformin 08(13.1\%) followed by Glimepiride +Metformin+pioglitazone 3(5.1\%), glimepride 1(1.7\%), tenegliptin $1(1.7 \%)$ and tenegliptin+Metformin 1(1.7\%). In the 29 anti-psychotic drugs, the most commonly prescribed drugs were chlordiazepoxide 6(9.8\%) and alprazolam 6(9.8\%) and the least commonly prescribed drugs were aripiprazole $1(1.7 \%)$ and phenobarbital $1(1.7 \%)$. The results are graphically represented in fig no. 3. 


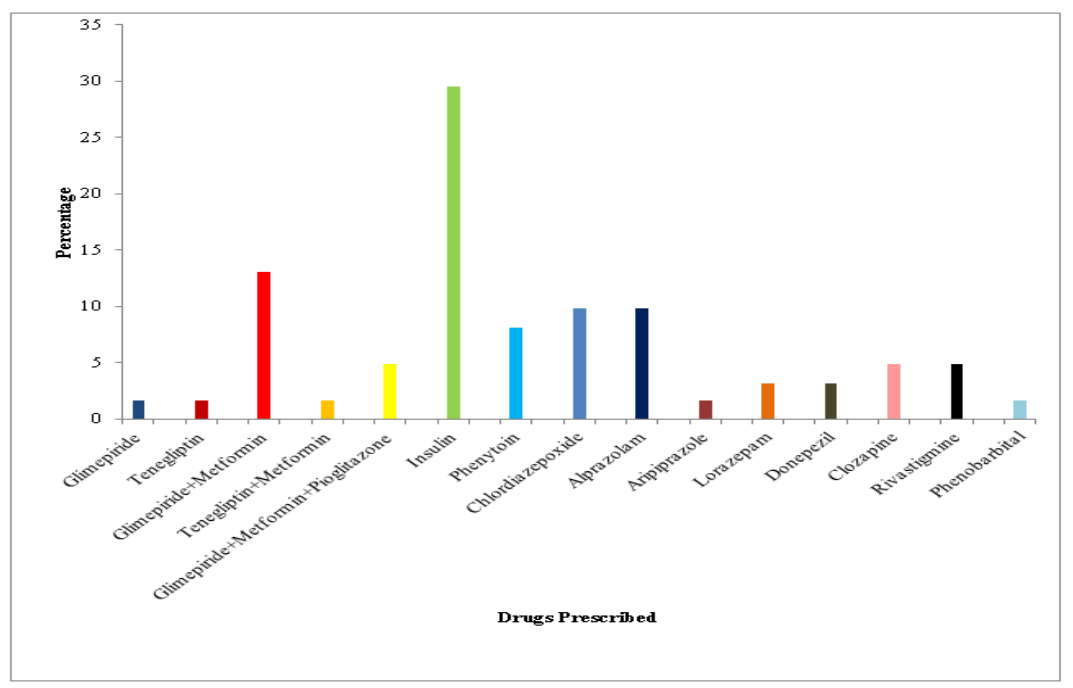

Figure 3: Prescription pattern of drugs

\section{Assessment of Efficacy}

On the basis of our study, efficacy of both antidiabetic and anti-psychotic drugs varied accordingly from subject to subject. For majority of the subjects, both drugs were effective, whereas it was less effective for a few. When the subjects were asked about how they feel after taking medications, 19 (70.4\%) answered that they are feeling better and $08(29.6 \%)$ replied that they are not.

\section{ADRs associated with the treatment}

A total of 27 subjects were enrolled in the study who were having psychiatric illness associated with DM. Among these subjects, no ADRs were observed and reported.

\section{DISCUSSION}

On analyzing our study, a total of 101 diabetic patients with or without co-morbid were enrolled. Among them, 27 in-patients were associated with psychiatric illness which was considered as comorbid condition. The prevalence of psychiatric illness with DM was found to be $26.7 \%$. The $p$-value is $p<0.0001$ which determines highly significant, hence according to this study; there is a strong relationship between psychiatric illness and diabetes.

A higher prevalence was noticed with age group 41-50 years. This is due to high perception of changes noticed by the patients during this age and their approach for the clinical diagnosis. According to the study, among the 27 subjects, high prevalence was noticed among the male gender. This may be due to the variation in population census or may be due to the increased health consciousness among males than females, or may be due to the lifestyle changes, whereas, Huang $\mathbf{C}$ et al., conducted a national population based cohort study on prevalence and incidence of mental illness in diabetes and found that the oneyear prevalence of mental illness among diabetic patients was $20.6 \%$ in 2000 and the cumulative prevalence increased to $42.2 \%$ in 2004 . In their study, a higher prevalence was noticed with age $\geq$ 45 , and with female gender. ${ }^{4}$

According to this study, the determinant factors for the psychiatric illness were found to be being women, having no income, being at the age of menopause, having no physical activity, being illiterate and were widowed or unmarried.

Being women is considered as a determinant factor since women spend most of their time at home, they are more emotional and they are having poor eating habits too. Their hormonal changes can also have an impact, which shows the disorders in women at the age of menopause. The study has also revealed that illiterate persons and those who are not having income are susceptible to psychiatric illness associated with DM. This may be due to the lack of knowledge and understanding about diabetes, and lack of social security. Being single can worsen the mental status of a person with increase in their age, and also having no physical activity can reduce the self management and thus can lead to poor glycemic control, while 
Sun $\mathbf{N}$ et al., conducted a similar study to evaluate the prevalence and determinants of anxiety and depression and to assess their impact on glycemic control in participants with type 2 diabetes mellitus and the determinant factors were found to be being woman, older age, low education level, being single, diabetes complications, anxiety symptoms, depressive symptoms, low income, chronic disease and poor sleep quality. ${ }^{2}$

In this study, among the anti-diabetic drug prescriptions, there was a higher percentage of use of insulin and the most commonly prescribed drug was combination of Glimepride+ Metformin (sulfonylurea and biguanide) which may be due to the high preference of these drugs by the physician. Agarwal A et al., conducted a study to assess the prescribing pattern and efficacy of anti-diabetic drugs in maintaining optimal glycemic levels in diabetic patients and they reported that sulfonylureas (for eg.,Glimepride) were the most commonly prescribed class, but metformin (biguanide) was the commonest prescribed individual drug among oral hypoglycemic agents (OHA). They also reported that fixed dose combination of biguanide and sulfonylurea was prescribed commonly and monotherapy dominated over polytherapy and there was a higher percentage of use of insulin in Type 2 diabetics. ${ }^{5}$

In accordance with the current study, the most commonly prescribed anti-psychotic drugs were chlordiazepoxide 6(9.8\%) and alprazolam 6(9.8\%) and the least commonly prescribed drugs were aripiprazole 1(1.7\%) and phenobarbital 1(1.7\%), whereas, Fourrier A et al., conducted a study on patterns of neuroleptic drug prescription and they reported that phenothiazines were the most often prescribed, followed by butyrophenones, benzamides, other neuroleptics and thioxanthenes 6

On the basis of our study, efficacy of both antidiabetic and anti-psychotic drugs varied accordingly from subject to subject. For majority of the subjects, both drugs were effective, whereas it was less effective for a few. When the subjects were asked about how they feel after taking medications, 19 (70.4\%) answered that they are feeling better and $08(29.6 \%)$ replied that they are not.
No ADRs were observed in the study, may be due to lack of awareness or due to lack of effective follow-up.

The study shows that, majority of the subjects rarely participated in any social activities and they were feeling upset about it. This may be due to their hesitation to be a part of social event since they feel anxious and nervous when they focus on their concerns regarding the chronic illness i.e., diabetes, which affects them physically, socially and mentally and makes them feel vulnerable, confused and worried about their health and future, which is again revealed when majority of the subjects responded that they are often discouraged by their health problems and most of them stated that they don't feel better about themselves, which shows their lack of selfconfidence. The study also revealed that majority of the subjects was having poor sleep quality, which indicates their consciousness regarding their health.

\section{CONCLUSION}

According to the analyzed results and from view of literature, the conclusions made are:

- $\quad$ Psychiatric illness is most prevalent in Diabetic patients. Anxiety is the most commonly seen psychiatric illness among diabetic patients.

- The common determinant factors of psychiatric illness in diabetic patients were being women, having no income, being at the age of menopause, having no physical activity, being illiterate and being single or widowed.

- Among the anti-diabetic drug prescriptions, there is a higher percentage of use of insulin and the most commonly prescribed drug is combination of Glimepride+ Metformin (sulfonylurea and biguanide). The most commonly prescribed antipsychotic drugs were chlordiazepoxide and alprazolam and the least commonly prescribed drugs were aripiprazole and Phenobarbital.

- Efficacy of both anti-diabetic and antipsychotic drugs varied accordingly from subject to subject. For majority of the subjects, both drugs were effective, whereas it was less effective for a few.

- The current study revealed a close connection of diabetes with psychiatric illness and the determinant factors associated with it. Hence it is propounded to conduct further studies on this 
Akza K Alex et al., Journal of Biomedical and Pharmaceutical Research

topic and to create awareness among the diabetic patients at prior stage itself to eradicate such a complication from the society.

\section{ACKNOWLEDGEMENT}

It is a pleasure and privilege to express my deep sense of thanks and indebtedness to the management of SJM College of Pharmacy Karnataka, Dr. Bharathi D.R, Principal SJM College of Pharmacy and Mr. Nataraj GR for giving the consent and facilities to carry out this work.

\section{REFERENCES:}

1. Balhara Y. Diabetes and psychiatric disorders. Indian J Endocrinol Metab 2011; 15(4):274.

2. Sun N, Lou P, Shang Y, Zhang P, Wang J, Chang $G$ et al. Prevalence and determinants of depressive and anxiety symptoms in adults with type 2 diabetes in China: a cross-sectional study. BMJ Open 2016;6(8):1-8.

3. Subramaniam M, Chong $S$, Pek E. Diabetes Mellitus and Impaired Glucose Tolerance in Patients with Schizophrenia. Can J Psychiatry 2003;48(5):345-47.

4. Huang $C$, Wang $S$, Lee $M$, Chiu $H$. Prevalence and incidence of mental illness in diabetes: $\mathrm{A}$ national population-based cohort study. Diabetes Res Clin Practice 2011;93(1):106-14.

5. Agarwal A, Jadhav P, Deshmukh Y. Prescribing pattern and efficacy of anti-diabetic drugs in maintaining optimal glycemic levels in diabetic patients. J Basic Clin Pharm 2014;5(3):79.

6. Fourrier A, Gasquet I, Allicar M, Bouhassira $M$, Lépine J, Bégaud B. Patterns of neuroleptic drug prescription: a national cross-sectional survey of a random sample of French psychiatrists. Br J Pharmacol 2001;49(1):80-86.

7. Surwit $R$, Schneider M, Feinglos $M$. Stress and Diabetes Mellitus. Diabetes Care 1992;15(10):1413-22.

8. Elamoshy R, Bird Y, Thorpe L, Moraros J. Risk of Depression and Suicidality among Diabetic Patients: A Systematic Review and MetaAnalysis. J Clin Med 2018;7(11):445.

9. Khandelwal D, Bahety $P$, Agarwal G, Dutta $D$, Kalra S, Taparia $P$ et al. Occurrence and predictors of depression and poor quality of life among patients with Type-2 diabetes: A Northern India perspective. Indian J Endocrinol Metab 2017;21(4):564.

10. AlBekairy A, AbuRuz $S$, Alsabani B, Alshehri A, Aldebasi T, Alkatheri A et al. Exploring Factors Associated with Depression and Anxiety among Hospitalized Patients with Type 2 Diabetes Mellitus. Medical Principles and Practice 2017;26(6):547-53.

11. Mossie T, Berhe G, Kahsay G, Tareke M. Prevalence of depression and associated factors among diabetic patients at Mekelle City, North Ethiopia. Indian J Psychol Med 2017; 39(1):52.

12. Golden $S$, Shah N, Naqibuddin M, Payne J, HillBriggs $F$, Wand $G$ et al. The Prevalence and Specificity of Depression Diagnosis in a ClinicBased Population of Adults With Type 2 Diabetes Mellitus. Psychosomatics 2017;58(1): 28-37. 